\title{
Necrotising sarcoid granulomatosis: clinical, functional, endoscopical and radiographical evaluations
}

\author{
C. Quaden*, I. Tillie-Leblond*, A. Delobbe*, L. Delaunois*", A. Verstraeten`, \\ M. Demedts ${ }^{+}$and B. Wallaert*
}

ABSTRACT: Necrotising sarcoid granulomatosis (NSG) is a rare disease diagnosed on the basis of pathological features. The present study reports the characteristics of 14 cases of NSG.

The mean age at the appearance of first symptoms was 37 yrs and the mean delay between first symptoms and diagnosis was 1 yr. Extrarespiratory symptoms were more common (12 out of 14) than respiratory symptoms (eight out of 14). Seven patients had inflammatory syndrome. Bronchoalveolar lavage was performed in eight patients and found to be normal in three cases. Respiratory function was normal in 13 patients, but carbon monoxide diffusing capacity was slightly decreased in eight of the 11 patients tested. A computed tomography scan showed a solitary nodule in four out of 14 cases, bilateral nodules in three and infiltrates in seven.

One patient died from neurological complications despite treatment with corticosteroids and immunosuppressive drugs. Two cases of relapse were observed in patients initially treated with corticosteroids, and there were two cases of relapse after surgery. No relapse occurred in the five untreated patients. During the follow-up, lung cancer was detected at 26 months and 8 yrs, respectively, after NSG diagnosis in two patients.

In conclusion, no one treatment is associated with a better outcome than the others, although lung biopsy might be necessary in case of isolated nodule or cavitation. Greater vigilance is required during the follow-up.

KEYWORDS: Granulomatosis, necrotising, sarcoid-like

$\mathbf{N}$ ecrotising sarcoid granulomatosis (NSG) is a rare systemic disease, characterised by sarcoid-like granuloma, vasculitis and variable degrees of necrosis. Since its first description in 1973 by LIEBOw [1], several additional series and some case reports have been published. The aetiology and pathogenesis are both unknown. Common histological features are associated with a variety of clinical presentations in a heterogeneous population. For this reason, it is uncertain whether the histological characteristics represent a disease entity or a nonspecific reaction to various triggers. The aim of the present study is to describe the clinical course of patients diagnosed with NSG after histological examination of lung specimens.

\section{MATERIALS AND METHODS}

\section{Patient selection and study design}

This multicentre study was conducted in five pulmonology departments at university hospitals (Lille and Roubaix, France; Mont-Godine, Gent and Leuven, Belgium) that treat patients with NSG. The centres were contacted by e-mail and asked to identify all patients diagnosed with NSG between January 1, 1989 and December 31, 2003. Patients were eligible if they presented pathological signs of NSG [1], namely numerous parenchymal sarcoid-like granulomas and noncaseating necrosis associated with granulomatous-, giant cell- or lymphocytic-type vasculitis involving both arteries and veins. Lung biopsies were all negative for bacteria, fungi and acid-fast bacilli (stains and cultures).

\section{AFFILIATIONS}

*Service de Pneumologie et d'Immuno-Allergologie, Hôpital Calmette, CHRU, Lille, France.

${ }^{\#}$ Service de Pneumologie, Cliniques Universitaires UCL, Mont-Godine,

'Service de Pneumologie, AZ-Saint Lucas, Gent, and

+Service de Pneumologie, Université Zickenhuizen, Leuven, Belgium.

CORRESPONDENCE

B. Wallaert

Service de Pneumologie et

d'Immuno-Allergologie

Hôpital Calmette

1 rue Leclerca

59037 Lille Cedex

France

Fax: 33320445768

E-mail: bwallaert@chru-lille.fr

Received:

March 022005

Accepted after revision: July 142005

European Respiratory Journal Print ISSN 0903-1936 Online ISSN 1399-3003 
Patients attended the pulmonology departments at least every 6 months. A standard form was used to record the characteristics of the patients. The form comprised the following items: 1) epidemiological characteristics (age, sex, smoking history, occupation); 2) clinical and functional parameters, and results of a computed tomography (CT) scan at initial evaluation; and 3) follow-up evaluation under treatment, i.e. clinical and functional parameters and results of the CT scan.

\section{Methods}

Biological methods

All patients were tested for serum markers of inflammation. The erythrocyte sedimentation rate (normal: $7-15 \mathrm{~mm}$ in first hour) and C-reactive protein (CRP) concentration (normal: 0-6 $\left.\mathrm{mg} \cdot \mathrm{L}^{-1}\right)$ were measured for all patients. Serum angiotensin-converting enzyme (ACE; normal: 10-55 IU $\cdot \mathrm{L}^{-1}$ ), antineutrophil cytoplasmic antibody (ANCA), antinuclear antibody and rheumatoid factor (as determined by laser nephelometry, where a titre of $\geqslant 40 \mathrm{IU}$ was considered positive) were determined in all cases.

\section{Pulmonary function tests}

The pulmonary function tests consisted of standard spirometry and measurement of lung volumes with a body plethysmograph. The following parameters were recorded: forced expiratory volume in one second, forced vital capacity, functional residual capacity, residual volume and total lung capacity. The carbon monoxide diffusing capacity of the lung $(D \mathrm{~L}, \mathrm{CO})$ was determined by the single-breath method [2] and corrected for haemoglobin level. Data are expressed as absolute values and percentages of the predicted values [3].

\section{Computed tomography}

CT was always performed in the supine position. The CT scans were reviewed retrospectively and were independently interpreted by both a chest radiologist (M. Rémy-Jardin) and a chest physician (I. Tillie-Leblond). Agreement between the two readers was obtained by reaching a consensus regarding the nature of pulmonary abnormalities. The interobserver correlation coefficient was 0.86 . Lesions seen on the CT scans were defined as solitary nodules, bilateral nodules, infiltrates, cavitation, hilar or mediastinal adenopathy and pleural thickening. Nodular lesions, characterised by soft tissue attenuation, consisted of micronodules $(<5 \mathrm{~mm})$ and larger nodules $(5-10 \mathrm{~mm}$ or $>10 \mathrm{~mm}$ ). Cavitated nodules (with a central hole) were randomly distributed among nodules.

\section{Bronchoscopy procedures}

Endobronchial exploration was performed in each patient using a fibreoptic bronchoscope as previously described [4]. The larynx was anaesthetised with a $2 \%$ Xylocaine ${ }^{\circledR}$ spray (AstraZeneca, Lund, Sweden). The degree of oxygen saturation was evaluated with a digital oxymeter. Macroscopic aspects of the tracheobronchial tree were recorded. Tracheobronchial secretions were sent for bacteriological and cytological examination. Patients also underwent bronchoalveolar lavage (BAL), bronchial biopsies and transbronchial biopsies (TBBs). BAL was performed according to standardised techniques [4] before biopsies were obtained to prevent alteration of cell values in the lavage fluid by blood leakage. When performed, TTBs were obtained by inserting forceps into a small peripheral bronchus. On average, five biopsies were obtained.

\section{RESULTS \\ Clinical features}

In total, 14 patients from five different pulmonary institutions were included in the study. Of these, 12 patients were followed by three centres. Two of these patients have been described previously $[5,6]$. Patients were followed-up for between 18 and 125 months (mean 56.6) at the time of final evaluation in December 2003.

Of the 14 patients, 10 were female and four were male (mean age: 42 yrs, range: 15-67) and all were Caucasian. The occupational and allergic history was noncontributive in all cases. Patients had previously had the following diseases: pulmonary tuberculosis $(n=2)$; type 2 diabetes $(n=2)$; thyroid disorder $(n=2)$; cancer of the oesophagus $(n=1)$; and idiopathic thrombocytopenia $(n=1)$. The clinical characteristics of the patients at the time of diagnosis are summarised in table 1. All patients except one were symptomatic at the time of the initial evaluation. Patient No. 11 was asymptomatic, but lung lesions were discovered by chance during a routine chest radiograph. The duration of symptoms prior to hospitalisation varied from several weeks to 1 yr. Extrarespiratory manifestations (86\%) were more frequent than respiratory manifestations (57\%). In

\begin{tabular}{|c|c|}
\hline Features & Patients \\
\hline \multicolumn{2}{|l|}{ Smoking habits } \\
\hline Nonsmokers & $9(64)$ \\
\hline Ex-smokers & $1(7)$ \\
\hline Smokers & $4(29)$ \\
\hline Respiratory manifestations & $8(57)$ \\
\hline Cough & $5(36)$ \\
\hline Dyspnoea & $4(29)$ \\
\hline Exertional & $4(29)$ \\
\hline At rest & $0(0)$ \\
\hline Chest pain & $4(29)$ \\
\hline Extrarespiratory manifestations & $12(86)$ \\
\hline Systemic symptoms & $8(57)$ \\
\hline Easily tired & $7(50)$ \\
\hline Fever & $5(36)$ \\
\hline Weight loss & $4(29)$ \\
\hline Night sweats & $2(14)$ \\
\hline Arthralgia & $5(36)$ \\
\hline Ocular manifestations & $3(21)$ \\
\hline Sicca syndrome & $2(14)$ \\
\hline Neurological symptoms & $1(7)$ \\
\hline Nasal symptoms & $1(7)$ \\
\hline Skin involvement & $1(7)$ \\
\hline \multicolumn{2}{|l|}{ Physical findings } \\
\hline Crackles & $1(7)$ \\
\hline Joint swelling & $1(7)$ \\
\hline Splenomegaly & $1(7)$ \\
\hline
\end{tabular}

Data are presented as n (\%) 
TABLE 2 Results of pulmonary function tests in 14 patients with necrotising sarcoid granulomatosis

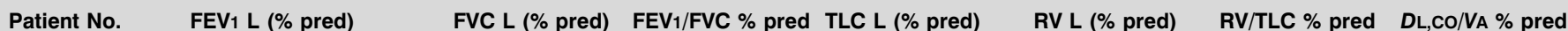

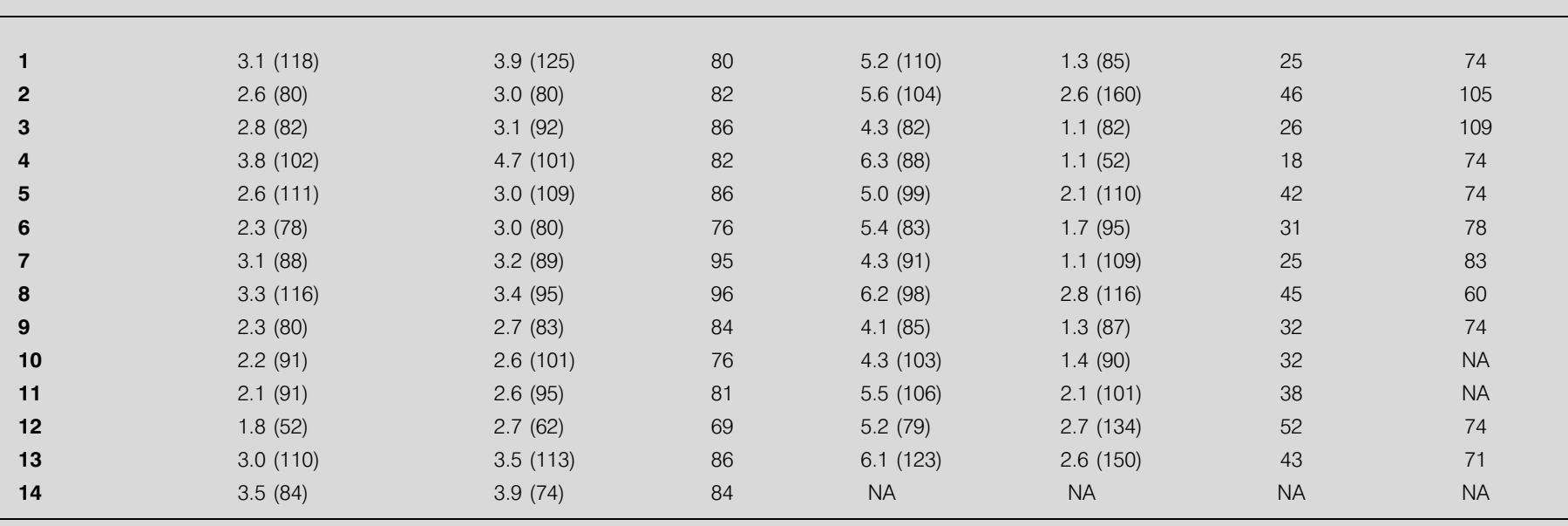

FEV1: forced expiratory volume in one second; \% pred: percentage of predicted; FVC: forced vital capacity; TLC: total lung capacity; RV: residual volume; DL,Co: carbon monoxide diffusing capacity of the lung; VA: alveolar volume; NA: not available.

$58 \%$ of cases, extrarespiratory manifestations first appeared at the same time as respiratory manifestations. In $29 \%$ of cases, extrarespiratory manifestations preceded pulmonary involvement by up to 2 yrs for cutaneous lesions in patient No. 14 . Nonpulmonary manifestations followed respiratory manifestations in $17 \%$ of cases, with a mean delay of $1 \mathrm{yr}$.

Two patients (patient Nos 5 and 10) presented with Sicca syndrome (SS) with keratoconjunctivitis and xerostomia (Schirmer's test; rose bengal staining of the cornea and minor salivary gland biopsies). The most severe extrarespiratory involvement occurred in patient No. 6 who, after chronic uveitis, developed paresis of the four limbs, cerebellar syndrome and cranial nerve palsy. This patient displayed multiple nodular lesions on encephalic magnetic resonance imaging. No brain biopsy was performed. Cerebral NSG was diagnosed after excluding other causes by routine exploratory tests, including examination of cerebrospinal fluid.

Pulmonary examination was normal except in patient No. 12, where crackles were observed in lower lung fields, correlated with radiographical evidence of pulmonary infiltrates.

\section{Inflammatory markers}

The erythrocyte sedimentation rate (ESR) was elevated in nine out of the 14 cases. The ESR was $>100 \mathrm{~mm} \cdot \mathrm{h}^{-1}$ in two cases (patient Nos 1 and 7). Other markers of inflammation (CRP and fibrinogen) were within the normal range. The level of ACE was normal in all cases. All subjects were negative for rheumatoid factor and ANCAs. Patient No. 5, who had SS, was positive for antinuclear antibodies with a high titre (one out of $10,240)$ and a SS-A and SS-B characterisation.

\section{Pulmonary function tests}

The pulmonary function test results of the 14 study patients are shown in table 2.

Lung volumes were normal in 13 out of 14 patients. An obstructive-restrictive ventilatory defect was present in one case (patient No. 12; regular smoker). This defect did not improve following treatment with a short-action $\beta_{2}$-mimetic. DL,CO was low for lung volume in eight of the 11 patients tested. This alteration was slight in most cases $(70-80 \%$ of normal in seven out of eight patients).

\section{CT findings}

Table 3 shows the CT findings in the patients with NSG.

The most common feature (seven out of 14 cases) was alveolar infiltrates (figs 1 and 2). This was particularly common in the subpleural area, without preference for any lobe (fig. 3). Air bronchogram was found in four of the seven subjects (patient Nos 1, 2, 7 and 9; fig. 3). Solitary nodules were detected in four out of the 14 cases and bilateral nodules with regular or slightly irregular margins in three cases (patient Nos 3, 13 and 14; fig. 4). The nodular disease was not particular associated with any one lobe. Nodules ranged from $2-4 \mathrm{~cm}$ in diameter. Cavitation was present in only two cases (fig. 4; patient Nos 11 and 12). Hilar or mediastinal adenopathy was detected in five cases (patient Nos 6, 7, 9, 10 and 12). These lymphadenopathies were not compressive (maximal diameter $2 \mathrm{~cm}$ in patient

\section{TABLE 3 Computed tomography findings in 14 patients} with necrotising sarcoid granulomatosis

\begin{tabular}{ll} 
Finding & Patients \\
\hline Infiltrates & $7(50)$ \\
Solitary nodule & $4(29)$ \\
Bilateral/multiple nodules & $3(21)$ \\
Cavitation & $2(14)$ \\
Hilar or mediastinal adenopathy & $5(36)^{\#}$ \\
Pleural thickening & $1(7)$ \\
\hline
\end{tabular}

Data are presented as $\mathrm{n}(\%) .{ }^{*}$ : bilateral in all cases. 


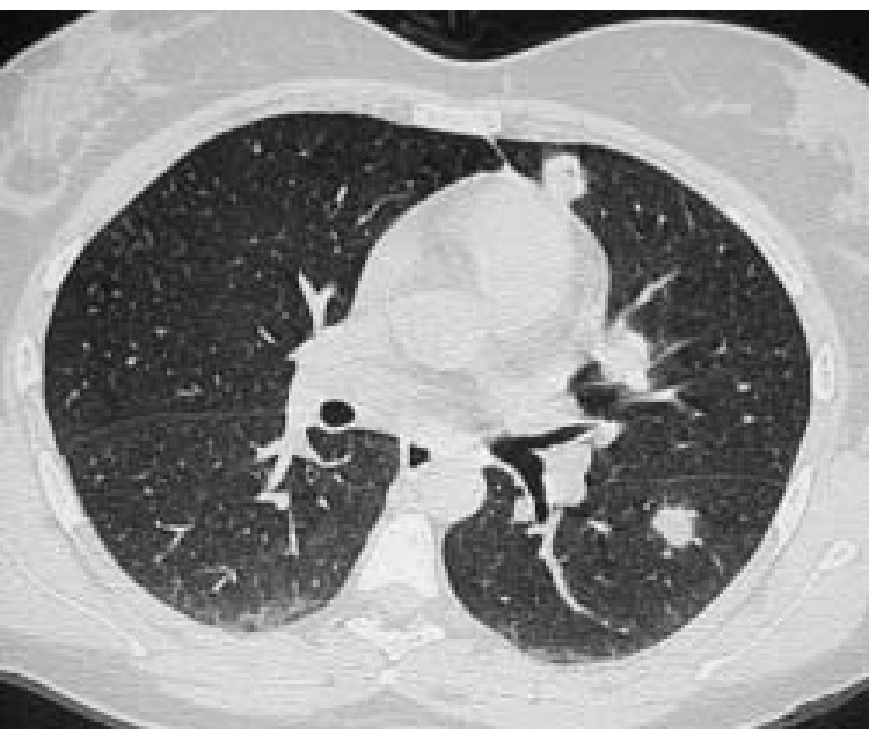

FIGURE 1. Computed tomography scan of the lower lung zones showing bilateral airspace consolidation.

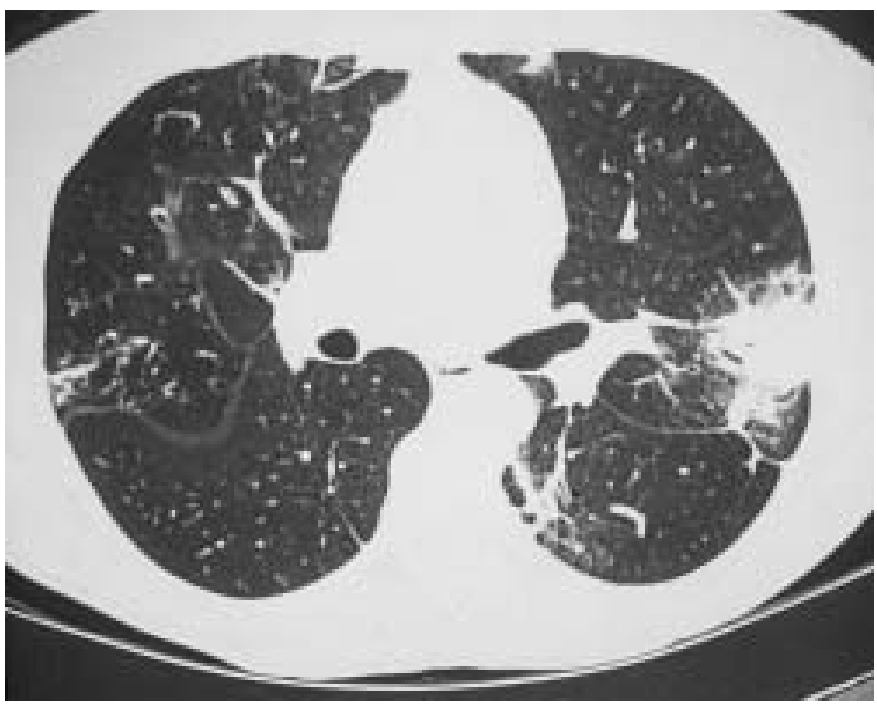

FIGURE 2. Computed tomography scan at the level of the right bronchus intermedius showing small airway disease in the right upper lobe with areas of airspace consolidation in the lingula and the apical segment of the left lower lobe. The peripheral location of the axillary mass with irregular borders should be noted.

No. 6) and were bilateral. Pleural thickening was noted in only two cases (patient Nos 1 and 10). It was minimal and located in front of a parenchymal nodule. There was no pleural effusion.

\section{Endoscopic and bronchoalveolar findings}

Macroscopic evaluation was normal in all cases. Cytological examination of the secretions revealed no atypical cells. The tracheobronchial secretions were always negative for bacteria, fungi and acid-fast bacilli. BAL was performed in eight patients (table 4), including one who was a regular smoker. Considering their smoking habits, BAL cell count and formula were normal in three cases (patient Nos 1,2 and 4). Six patients displayed an elevated number of total recovered cells.

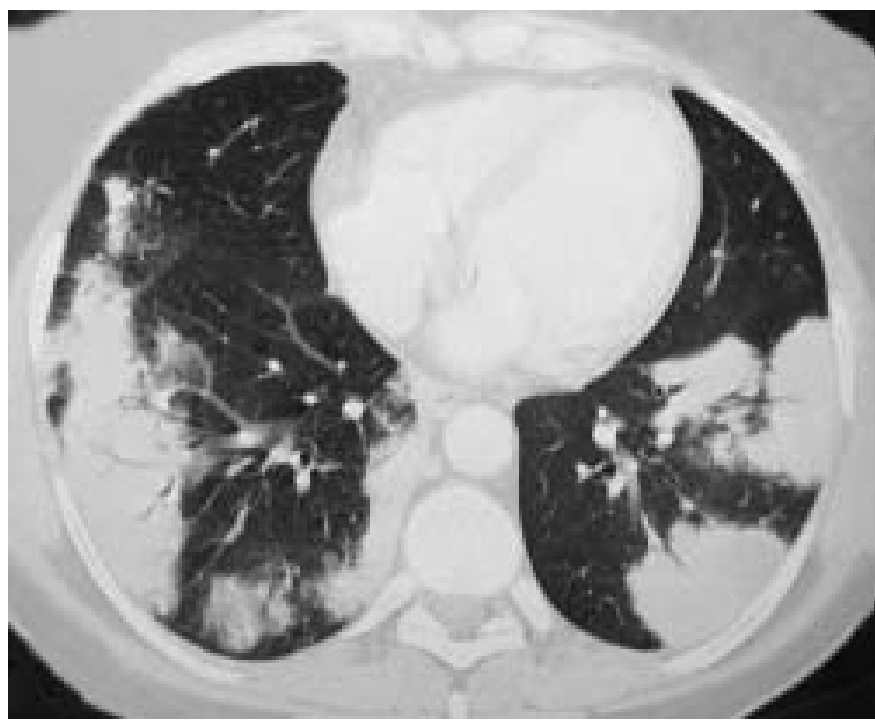

FIGURE 3. Computed tomography scan at the level of the right bronchus intermedius, showing three nodular opacities with irregular borders, one located in the lingula and one located in the left lower lobe. The presence of areas of groundglass attenuation in the posterior parts of both lower lobes corresponds to hypoventilated lung.

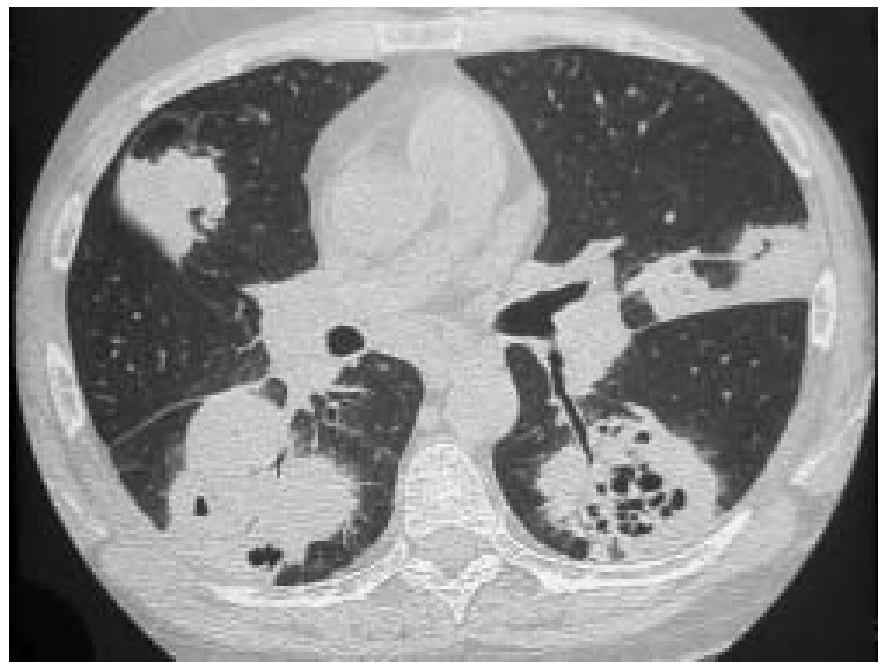

FIGURE 4. Computed tomography scan at the level of the right bronchus intermedius, showing large areas of airspace consolidation in the right middle lobe, lingula and the apical segments of both lower lobes. The presence of cavitation within the right and left lower lobe masses should be noted.

Neutrophil percentage was elevated in two patients, eosinophil percentage in one and lymphocyte percentage in two. In the two patients with an elevated lymphocyte percentage, the CD4+/CD8+ ratio was 0.9 and 1.4 (patient Nos 7 and 10, respectively). Pigment-laden macrophages were in the normal range. Bronchial biopsies and TBBs were performed in patient Nos 3 and 4, respectively. The pathological findings are summarised as follows. Abnormal BAL total cell counts or formulae could be found in any of the three major presentations. 


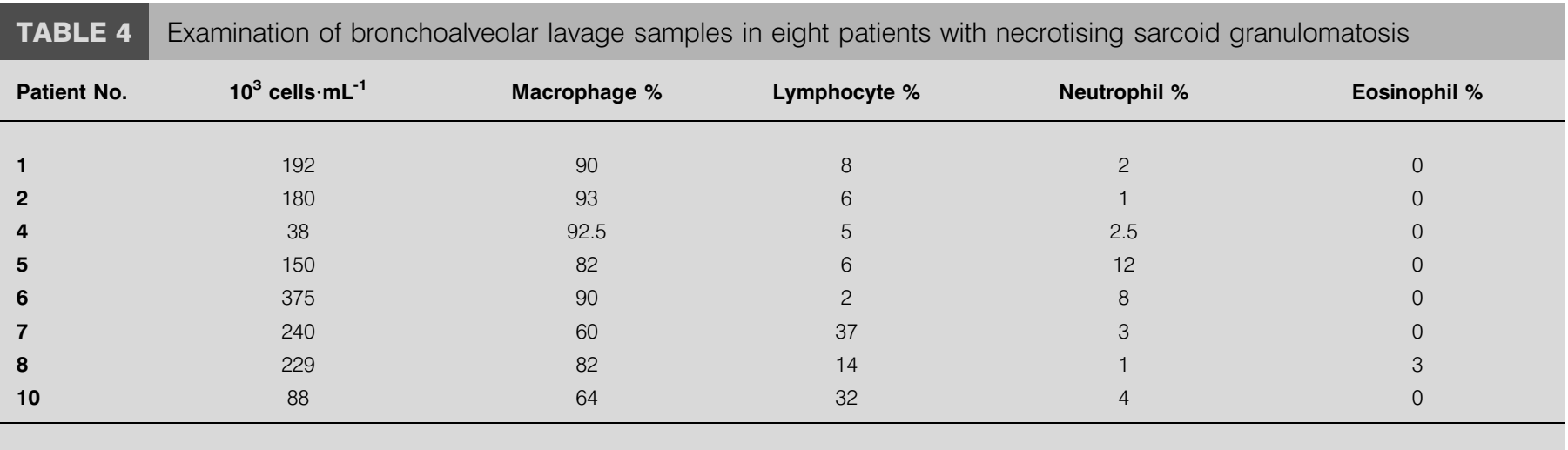

\section{Pathological findings}

As NSG is characterised by distinct histopathological features, definitive diagnosis depends on correct pathological examination of biopsy specimens. In the present study, material was used from surgical procedures in all cases (figs 5 and 6).

Bronchial biopsies were performed before lung biopsies in three cases (patient Nos 3, 5 and 10). A sarcoid granuloma was found in one case (patient No. 10), whereas the other pathological features characteristic of NSG, namely the presence of vasculitis and necrosis, were absent. TBBs were performed in four patients (patient Nos 1, 5, 6 and 12) who had diffuse infiltrates. They were normal in two cases (patient Nos 5 and 6). Patient No. 1 was diagnosed with organising pneumonia. Patient No. 12 presented with epithelioid granulomatous inflammation. Transthoracic fine-needle aspiration was performed in four patients. Nonspecific inflammation was found in three cases (patient Nos 5, 11 and 13). A sarcoid granuloma was found in patient No. 6, but no angiitis or necrosis was observed. Subsequently, patient No. 6 underwent mediastinoscopy, revealing nonspecific inflammatory infiltration of the lymph node. Examination of biopsies from extrarespiratory localisations did not lead to the diagnosis of NSG. Minor salivary gland biopsies were performed in two

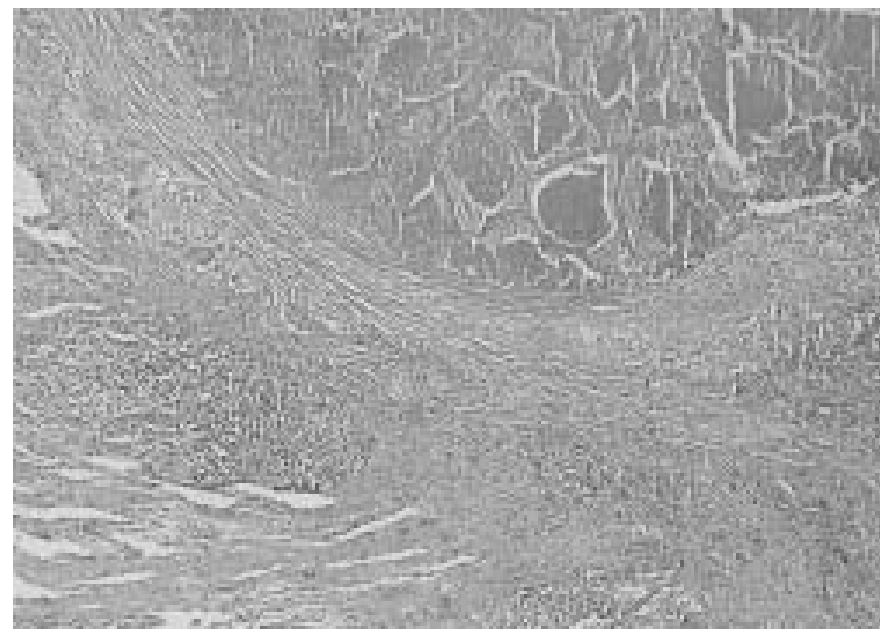

FIGURE 5. Histological stain showing coagulative necrosis and individual granulomas. cases. In patient Nos 5 and 10, who both had SS, these biopsies revealed lymphocytic inflammatory infiltration associated with sarcoid granuloma. No necrosis or angiitis were detected in one case. A nasal biopsy taken from patient No. 10 during an episode of rhinitis was not contributive. A skin biopsy from patient No. 14, who presented facial dermatosis, showed inflammatory changes with one epithelioid granuloma.

In this study, diagnosis of NSG was obtained by thoracotomy in all cases with 10 open-lung biopsies, two segmentectomies, one lobectomy and one wedge resection. This was the main inclusion criterion. Microscopic examination revealed a combination of granulomas (figs 5 and 6), necrosis (fig. 5) and angiitis in all specimens. The background lesion consisted of numerous parenchymal granulomas that were always sarcoidlike in nature. These isolated or confluent granulomas were hyalinised in some foci. Several kinds of vascular lesions were observed, as described by LIEBOW [1]. Angiitis generally consisted of intramural granulomas or diffuse lymphocitic and plasma cell infiltration more or less confined to vessel walls. Giant cell arteritis was less frequent. The extent of coagulative necrosis varied considerably, from small punctate foci in angiocentric granulomas to large extravascular confluent zones. Pleural thickening was found in two cases.

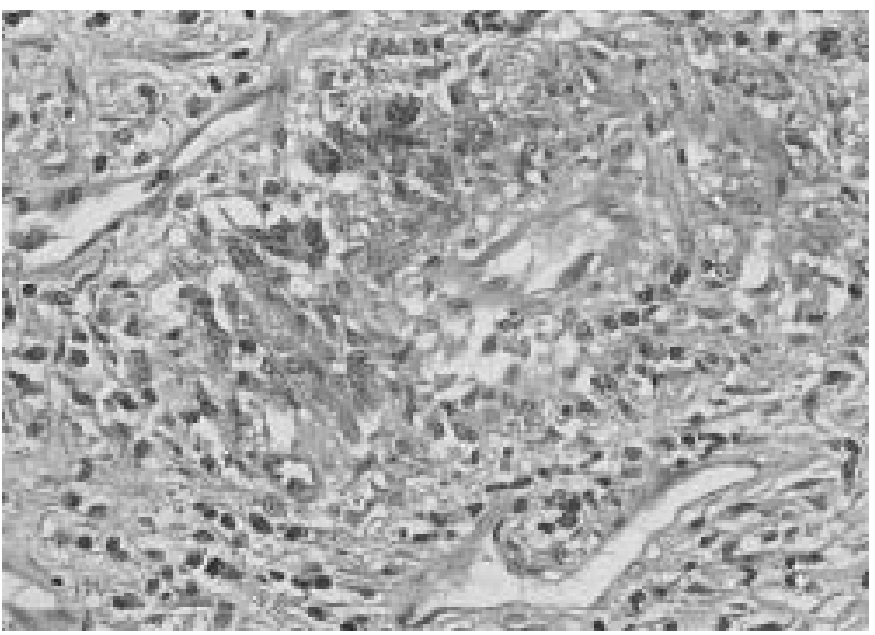

FIGURE 6. Histological stain showing vascular involvement with extensive granulomatous infiltration of the media. 
TABLE 5 Treatment and clinical course in 14 patients with necrotising sarcoid granulomatosis

\begin{tabular}{lcccc} 
Patient No. & Treatment & Indication & Respiratory course & Extrarespiratory course \\
\hline $\mathbf{1}$ & Steroid & Systemic symptoms & Resolution relapse & Resolution \\
$\mathbf{2}$ & Steroid & Arthralgia & Resolution relapse & Persistent arthralgia \\
$\mathbf{3}$ & None & & Resolution & Resolution \\
$\mathbf{4}$ & Surgery & Diagnostic procedure & Resolution & Resolution \\
$\mathbf{5}$ & Steroid & Systemic symptoms & Resolution relapse & Resolution \\
$\mathbf{6}$ & S, C A, El & Cerebral involvement & Resolution & Death (neurological) \\
$\mathbf{7}$ & Steroid & Systemic symptoms & Resolution & Resolution \\
$\mathbf{8}$ & Surgery & Diagnostic procedure & Relapse of nodule & None \\
$\mathbf{9}$ & None & & Resolution & Resolution \\
$\mathbf{1 0}$ & Surgery & Diagnostic procedure & Relapse of nodules & Pericarditis C-myopathy \\
$\mathbf{1 1}$ & Surgery & Diagnostic procedure & Resolution & None \\
$\mathbf{1 2}$ & None & & Sequelar cavities & Resolution \\
$\mathbf{1 3}$ & None & & Resolution & Resolution \\
$\mathbf{1 4}$ & None & & Resolution & 36 \\
\hline
\end{tabular}

S: steroid; C: cyclophosphamide; A: azathioprine; El: encephalic irradiation. ${ }^{\#}$ : neoplasy occurrence; " : aspergilloma and lung cancer.

\section{Treatment and clinical course}

The treatment and clinical course of the 14 patients are summarised in table 5. Mean follow-up from onset of disease to the end of the study was 54.6 months (range 18-114).

Five patients $(36 \%)$ initially received no treatment, and, of these, four (patient Nos 3, 9, 13 and 14) had a favourable course, clearing or considerably diminishing in 3-6 months with no relapse. The remaining patient (patient No. 12) presented with haemoptysis 26 months after NSG diagnosis. This was associated with pulmonary cavitary condensations and sequelae such as left cavities with aspergilloma. Bronchial biopsies revealed a squamous carcinoma of tumour, node, metastasis staging (cT1NOM0) and a right upper lobectomy was performed. This patient died from massive haemoptysis 3 months after the surgical procedure.

Five (36\%) patients (patient Nos 1, 2, 5, 6 and 7) initially received corticosteroids after NSG diagnosis, with starting doses of between 0.5 and $1 \mathrm{mg} \cdot \mathrm{kg}^{-1} \cdot$ day $^{-1}$ of prednisone or prednisolone. Indications for treatment are summarised in table 5 . The duration of steroid therapy ranged 1-68 months, with an average of 22.5 months. Steroids led to clearance of pulmonary condensations in 2-3 months in all five cases. Systemic symptoms (patient Nos 1, 5 and 7) and arthralgia resolved in $<3$ months under steroid therapy. Four patients responded favourably to steroids (patient Nos 1, 2, 5 and 7) and their treatment was progressively reduced. Patient No. 5 had a relapse when the prednisone dose was reduced from 30 to $20 \mathrm{mg} \cdot \mathrm{day}^{-1}$. The manifestations were the same as the first episode and responded to an increased steroid dose. This patient displayed steroid dependence, leading to prolonged treatment (68 months). Steroid therapy could be progressively reduced in patient Nos 1, 2 and 7 with no relapses. After discontinuation of treatment, a relapse occurred in patient No. 1. This patient presented the same clinical manifestations as during the first episode (cough and arthralgia), but different radiographic findings with bilateral nodules rather than irregular infiltrates. Again, these responded to steroid therapy. Eight years after NSG diagnosis, a right lower pulmonary mass (2.5 cm diameter) was detected in patient No. 2 and diagnosed as adenocarcinoma. In the 5th patient (patient No. 6), who displayed cerebral manifestations, corticosteroids were ineffective and immunosuppressive agents were required. Despite treatment with cyclophosphamide (13 months) and azathioprine $(76$ months), the disease progression was unfavourable. Encephalic irradiation ( $35 \mathrm{~Gy}$ ) failed to improve neurological degradation and the patient finally died 114 months after diagnosis.

The four patients $(36 \%)$ with solitary nodules underwent surgical resection. Relapse occurred in two cases. Patient No. 8 presented another solitary nodule 31 months after initial surgery; this nodule spontaneously disappeared after a 1-yr observation period. In patient No. 10, the radiological presentation of the relapse was different from that of the first episode, with numerous bilateral nodules detected 2 yrs after initial diagnosis of NSG. These nodules partially regressed in 3 months.

On analysing the NSG relapses more closely, the following features were noted: 1) initial therapy (steroid therapy or surgical resection) in all cases; 2) mean interval between treatment and relapse of 32 months (range 24-43); 3) clinical and radiological presentation identical to first episode in two and one case out of three, respectively; 4) spontaneously favourable progression in two out of three cases and good response to steroid therapy in one out of three cases; and 5) no initial biological, radiological, functional or BAL profiles were predictive of relapse.

\section{DISCUSSION}

In 1973, LIEBOW [1] made the first reference to a disease characterised by sarcoid-like granulomata with vasculitis and necrosis when describing his classification of pulmonary angiitis and granulomatosis not produced by known infectious 
agents or associated with rheumatoid disease. He named this disease "necrotising sarcoid granulomatosis" and initiated the debate as to whether the disease represented necrotising angiitis with sarcoid reaction or sarcoidosis with necrosis of the granuloma and the vessels. NSG is diagnosed on the basis of pathological features and shares common histological and clinical patterns with sarcoidosis.

Since the original description, six additional series have been published [7-12], along with some case reports, giving a total of $\sim 100$ cases. Extrarespiratory manifestations (mainly ophthalmological and neurological) $[5,7-9,13-15]$ and a globally benign course $[1,7-9,12,16-20]$ were described. The aim of the present study was to reconsider the major aspects of NSG, using respiratory exploratory tests that have not always been extensively evaluated in previous series.

The sex and age distributions agree with previous series. Most patients were nonsmokers (nine out of 14). Clinical presentation was variable and nonspecific. Interestingly, extrarespiratory manifestations were found to be more common than respiratory ones. Systemic symptoms were more common than previously reported by CHURG [9] and were prominent in most cases. Unlike subjects with Wegener's granulomatosis, patients with NSG have no upper airway disease, nephritis or systemic vasculitis [21]. In the same way, uveitis or cutaneous lesions are rare in NSG, contrasting with their frequency in sarcoidosis.

Limited information is available in the literature about laboratory tests in NSG patients. It was noted that inflammatory markers were elevated in most patients. Interestingly, ACE concentrations were normal in all patients, whereas they are elevated in $\sim 60 \%$ of patients with active sarcoidosis [19, 20]. Another biological parameter that could be useful for differential diagnosis was ANCA, as all tested patients in the study were negative for ANCA. ANCAs are clearly associated with systemic vasculitis (e.g. Wegener's syndrome), which is characterised by similar systemic symptoms and radiological patterns to NSG. Auto-antibodies (SS-A and SS-B) were detected in one patient with SS. None of the subjects in the study were positive for ACE or ANCA, which may be helpful when patients test positive for sarcoidosis or Wegener's syndrome.

Pulmonary function tests were normal in 13 out of the 14 patients. Only one patient had an obstructive-restrictive ventilatory defect with clinical evidence of coexisting chronic obstructive pulmonary disease. This contrasts with the results obtained by CHIтTоск et al. [7], who reported ventilatory defects in five out of seven patients: four restrictive patterns and one mixed restrictive-obstructive pattern. Another difference with the observation made by CНIтTOCK et al. [7] is the frequency of diffusing capacity alteration. DL,CO was decreased in eight out of 11 patients in the current study, whereas CНITTOCK et al. [7] observed a defect in only two out of seven cases. CT scan results are rarely described in NSG patients, as most previous large series were published before this technique was routinely used. Three main parenchymal CT features are typically reported: diffuse infiltrates, bilateral nodules and solitary nodules [7, 21]. The current results are in agreement with those of the study by CHIтTOCK et al. [7]. There is some debate as to whether hilar or mediastinal adenopathies are a feature of NSG. In the earliest first reports, in which chest radiograph was the only form of radiographical exploration, lymph node enlargement was considered to be uncommon and thought to represent a means of distinguishing NSG from sarcoidosis [10]. СНIтTOCK et al. [7] demonstrated that adenopathies were frequently found when CT scans were used (five out of seven patients). Bilateral hilar and/or mediastinal adenopathy were found in $36 \%$ of the study patients. Conversely, it was noted that pleural involvement was rare, whereas pleural thickening or effusion was seen on CT scans in six out of the seven patients reported by CHITTOCK et al. [7]. In the study by CHITTOCK et al. [7], the main symptom of each patient was pleuritic chest pain. Due to the major differences in the radiological features typical of sarcoidosis and NSG, and due to the difficulty associated with distinguishing between infectious granulomatous processes, tumours and Wegener's syndrome on CT scans (even when a bronchoscopic biopsy reveals a granuloma or if biological tests and BAL findings are negative), a lung biopsy is usually performed [21]. Although granulomas occur in a juxtalymphatic distribution which should mimic that seen in sarcoidosis, two important features of NSG distinguish it from sarcoidosis: the lower prevalence of mediastinal and hilar lymphadenopathy, and the cavitation seen in pulmonary lesions [21].

The value of BAL analysis when assessing lung involvement in NSG is unknown. The present study is the first to include the results of BAL explorations in NSG patients. It was noted that three of the eight patients tested had normal BAL findings. The others had variable and nonspecific features, associated with a predominance of lymphocytes, eosinophils or neutrophils. There was no alveolar haemorrhage.

As the histopathological features of NSG have been largely described previously [1, 8-10, 17, 22], the different techniques used to diagnose NSG were examined. In the current study, only large lung biopsies provided specimens that met these criteria. However, transbronchial needle aspiration (with or without ultrasound guidance) might be accurate in the diagnosis of benign lymphadenopathy including sarcoidosis. In addition, a needle biopsy of the parenchymal lesions might suggest the diagnosis of NSG, since Wegener's granuomatosis would be highly unlikely with a negative ANCA. Neoplastic lesions are unlikely in the absence of tumoural cells and a diagnosis of tuberculosis unlikely in the absence of organisms, since organisms are present in very high concentratiosn in the wall of a cavity or in an area of granulomatous necrosis. Therefore, it is likely that the diagnosis of NSG might be suspected and does not necessarily require thoracoscopic biopsy.

The data concerning the nature of the disease and its relationship to sarcoidosis are controversial. It is believed that clinical (frequency and severity of systemic symptoms), biological (normal ACE levels) and specific histopathological data are sufficient to show that NSG is a separate entity, slightly different from sarcoidosis. NSG appears to be intermediate between sarcoidosis and systemic necrotising vasculitides. Although NSG shares some pathological features with necrotising granulomatous angiitides, such as Wegener's granulomatosis or Churg-Strauss disease, it is not associated 
with ANCA, renal involvement or asthma. It is important to be able to distinguish NSG from these conditions because of differences in prognosis and response to treatment as demonstrated here.

The prognosis of NSG patients was described as being favourable in previous reports. In this study, only one patient had severe neurological complications leading to death. No other deaths related to central nervous system involvement have been reported in NSG patients. Two patients developed lung carcinoma 2 and 8 yrs after NSG diagnosis, respectively. NSG has previously been described in one patient with an oatcell carcinoma of the lung [23]. These two cases of lung cancer imply that a long observation period is required in patients with NSG and that the appearance of any new pulmonary condensations must be considered with great caution. In both cases, cancer was diagnosed on bronchial biopsy. In the remaining cases, treatment seemed to be associated with relapse and should be indicated only when symptoms are major.

In conclusion, given the relative benign nature of necrotising sarcoid granulomatosis, bronchoscopy should be performed to rule out cancer or infection, and lung biopsy is necessary in case of solitary nodule and/or cavitation (mainly when antineutrophil cytoplasmic antibodies are negative). Severe specific complications are rare in necrotising sarcoid granulomatosis, identified in only one case in this study. Contrasting with other studies, extrapulmonary manifestations can be found to be frequent in necrotising sarcoid granulomatosis. Relapses seem to be more frequent when treatment is proposed, and corticosteroids and other immunosuppressive drugs should be restricted to patients with major symptoms.

\section{ACKNOWLEDGEMENTS}

The authors would like to thank M-C. Coppin, M. RémyJardin, F. Steenhouwer, C. Tulippe and L. Delaunois for their contribution and constructive comments.

\section{REFERENCES}

1 Liebow AA. The J. Burns Amberson Lecture pulmonary angiitis and granulomatosis. Am Rev Respir Dis 1973; 108: $1-18$.

2 American Thoracic Society. Single-breath carbon monoxide diffusing capacity (transfer factor): recommendations for a standard technique-1995 update. Am J Respir Crit Care Med 1995; 152: 2185-2198.

3 Quanjer PH. Standardized lung function testing. Report working party, standardization of lung function tests. Bull Eur Physiopathol Respir 1983; 19: 1-95.

4 Technical recommendations and guidelines for bronchoalveolar lavage (BAL). Report of the European Society of Pneumology Task Group. Eur Respir J 1989; 2: 561-585.

5 Brevet F, Hachulla E, Courtin P, et al. Forme systémique de granulomatose nécrosante sarcoïdosique avec atteinte oculaire, pulmonaire et cérébrale [Systemic form of sarcoid-like necrotizing granulomatosis with ocular, pulmonary and cerebral involvement]. Rev Méd Interne 1993; 14: 243-248.

6 Radenne F, Tillie-Leblond I, Maurage CA, et al. Syndrome de Gougerot-Sjögren et granulomatose nécrosante sarcoïdosique [Sjogren's syndrome and necrotizing sarcoid-like granulomatosis]. Rev Mal Respir 1999; 16: 554-557.

7 Chittock DR, Joseph MG, Paterson NAM, et al. Necrotizing sarcoid granulomatosis with pleural involvement. Clinical and radiographic features. Chest 1994; 106: 672-676.

8 Churg A, Carrington CB, Gupta R. Necrotizing sarcoid granulomatosis. Chest 1979; 76: 406-413.

9 Churg A. Pulmonary angiitis and granulomatosis revisited. Hum Pathol 1983; 14: 868-883.

10 Koss MN, Hochholzer L, Feigin DS, et al. Necrotizing sarcoid-like granulomatosis: clinical, pathologic and immunopathologic findings. Hum Pathol 1980; 11: 510-519.

11 Sadoun D, Kambouchner M, Tazi A. Granulomatose nécrosante sarcoid-like [Necrotizing sarcoid granulomatosis. Apropos of 4 cases]. Ann Med Interne 1994; 145: 230-233.

12 Saldana MJ. Necrotizing sarcoid granulomatosis: clinicopathologic observations in 24 patients. Lab Invest 1978; 38: 364.

13 Beach RC, Corrin B, Scopes JW, et al. Necrotizing sarcoid granulomatosis with neurological lesions in a child. J Pediatr 1980; 97: 950-953.

14 Dykhuizen RS, Smith CC, Kennedy MM, et al. Necrotising sarcoid granulomatosis with extrapulmonary involvement. Eur Respir J 1997; 10: 245-247.

15 Singh N, Cole S, Krause PJ, et al. Necrotizing sarcoid granulomatosis with extrapulmonary involvement. Am Rev Respir Dis 1981; 124: 189-192.

16 Gibbs AR, Jones Williams W. Necrotizing sarcoidal granulomatosis: a problem of identity. Sarcoidosis 1987; 4: 94-96.

17 Niimi H, Hartman TE, Müller NL. Necrotizing sarcoid granulomatosis: computed tomography and pathologic findings. J Comput Assist Tomogr 1995; 19: 920-923.

18 Spiteri MA, Gledhill A, Campbell D, et al. Necrotizing sarcoid granulomatosis. Br J Dis Chest 1987; 81: 70-75.

19 Lieberman J, Nosal A, Schlessner LA, et al. Serum angiotensin converting enzyme for diagnosis and therapeutic evaluation of sarcoidosis. Am Rev Respir Dis 1979; 120: 329-335.

20 Rohatgi PK. Serum angiotensin converting enzyme in pulmonary disease. Lung 1982; 160: 287-301.

21 Frazier AA, Rosado-de-Christenson ML, Galvin JR, et al. Pulmonary angiitis and granulomatosis: radiologic-pathologic correlation. Radiographics 1998; 18: 687-710.

22 Saldana MJ, Israel HL. Necrotizing sarcoid granulomatosis, benign lymphocytic angiitis and granulomatosis: do they exist? Sem Respir Med 1989; 10: 182-188.

23 Stephen JG, Braimbridge MV, Corrin B, et al. Necrotizing sarcoidal angiitis and granulomatosis of the lung. Thorax 1976; 31: 356-360. 